\title{
Gamma Knife Radiosurgery for Metastatic Brain Tumors with Exophytic Hemorrhage
}

\author{
Eun Suk Park, M.D., Eun Jung Lee, M.D., Jung-Ho Yun, M.D., ${ }^{3}$ Young Hyun Cho, M.D., Jeong Hoon Kim, M.D., ${ }^{4}$ Do Hoon Kwon, M.D. \\ Department of Neurosurgery, Ulsan University Hospital, University of Ulsan College of Medicine, Ulsan, Korea \\ Department of Neurosurgery, ${ }^{2}$ Dongtan Sacred Heart Hospital, College of Medicine, Hallym University, Hwaseong, Korea \\ Department of Neurosurgery, ${ }^{3}$ Dankook University College of Medicine, Cheonan, Korea \\ Department of Neurological Surgery, ${ }^{4}$ Asan Medical Center, University of Ulsan College of Medicine, Seoul, Korea
}

Objective : Metastatic brain tumors (MBTs) often present with intracerebral hemorrhage. Although Gamma Knife surgery (GKS) is a valid treatment option for hemorrhagic MBTs, its efficacy is unclear. To achieve oncologic control and reduce radiation toxicity, we used a radiosurgical targeting technique that confines the tumor core within the hematoma when performing GKS in patients with such tumors. We reviewed our experience in this endeavor, focusing on local tumor control and treatment-associated morbidities.

Methods : From 2007 to 2014, 13 patients with hemorrhagic MBTs were treated via GKS using our targeting technique. The median marginal dose prescribed was 23 Gy (range, 20-25). GKS was performed approximately 2 weeks after tumor bleeding to allow the patient's condition to stabilize.

Results : The primary sites of the MBTs included the liver $(n=7)$, lung $(n=2)$, kidney $(n=1)$, and stomach $(n=1)$; in two cases, the primary tumor was a melanoma. The mean tumor volume was $4.00 \mathrm{~cm}^{3}$ (range, $\left.0.74-11.0\right)$. The mean overall survival duration after GKS was 12.5 months (range, 3-29), and three patients are still alive at the time of the review. The local tumor control rate was $92 \%$ (tumor disappearance $23 \%$, tumor regression $46 \%$, and stable disease $23 \%$ ). There was one (8\%) instance of local recurrence, which occurred 11 months after GKS in the solid portion of the tumor. No GKS-related complications were observed.

Conclusion : Our experience shows that GKS performed in conjunction with our targeting technique safely and effectively treats hemorrhagic MBTs. The success of this technique may reflect the presence of scattered metastatic tumor cells in the hematoma that do not proliferate owing to the inadequate microenvironment of the hematoma. We suggest that GKS can be a useful treatment option for patients with hemorrhagic MBTs that are not amenable to surgery.

Key Words : Neurosurgical procedures · Cerebral hemorrhage · Neoplasm metastases · Radiosurgery.

\section{INTRODUCTION}

Metastatic brain tumors (MBTs) are the most common adult brain tumors, occurring at an approximately 10 -fold greater rate than that of primary brain tumors ${ }^{16}$. The inci- dence of MBTs continues to increase along with advances in diagnostic imaging and systemic disease control. According to a review of large population- and autopsy-based studies, approximately $9-17 \%$ of cancer patients develop symptomatic MBTs $^{4)}$. The presenting symptoms of MBTs include head-

- Received : March 15, 2017 •Accepted : October 23, 2017

- Address for reprints : Do Hoon Kwon, M.D.

Department of Neurological Surgery, Asan Medical Center, University of Ulsan College of Medicine, 88 Olympic-ro 43-gil, Songpa-gu, Seoul 05505, Korea

Tel : +82-2-3010-3550, Fax : +82-2-476-6738, E-mail : ykwon@amc.seoul.kr

This is an Open Access article distributed under the terms of the Creative Commons Attribution Non-Commercial License (http://creativecommons.org/licenses/by-nc/4.0) which permits unrestricted non-commercial use, distribution, and reproduction in any medium, provided the original work is properly cited. 
aches, seizures, neurologic impairments, and in $3-14 \%$ of cases, tumoral hemorrhage, most commonly in metastases from renal cell carcinomas, choriocarcinomas, and melanomas ${ }^{1)}$. When MBTs present with hemorrhage, surgery is the first treatment option, especially in cases accompanying the mass effect. However, in cases that do not necessarily require open surgery, Gamma Knife radiosurgery (GKS) can be a reasonable alternative.

When we treated hemorrhagic MBTs via GKS, the large volume of the hematoma hindered the planning process, which included consideration of radiation toxicity. Moreover, the role of the scattered tumor cells in the hematoma in terms of tumor growth is obscure. Because we were uncertain whether our routine planning process targeted the tumor including the hematoma, we developed a radiosurgical targeting technique that confines the tumor core within the hematoma. We hypothesized that our technique would efficiently reduce radiation toxicity and achieve oncologic control. Herein, we review our experience using this planning technique, focusing on local tumor control and treatment-associated morbidities.

\section{MATERIALS AND METHODS}

\section{Patients}

From December 2007 to June 2014, 1645 patients received GKS for treatment of MBTs at our institution. The MBTs in 13 of these patients were accompanied by hematomas, and our targeting technique was used when performing GKS in these patients. All 13 patients had histologically confirmed primary cancers in extracranial lesions, presented with hemorrhagic brain metastases, and received the best medical treatment without surgery during the acute stage of hemorrhage. Brain metastases were diagnosed when brain magnetic resonance imaging (MRI) showed homogeneous contrast enhancement and different signal intensities in the hemorrhagic portion of the tumor (suggestive of a hematoma) versus the contrast-enhancing solid mass portion.

\section{Radiosurgical technique}

All patients were treated by using a Leksell Gamma Knife (model C or Perfexion) device (Elekta Instruments AB, Stockholm, Sweden). We first applied a stereotactic frame (Elekta Instruments $\mathrm{AB}$ ) to the patient's head after the patient had re- ceived a local anesthetic and an oral sedative. We then performed high-resolution, contrast-enhanced brain MRI and computed tomography (CT) for treatment planning purposes. Treatment plans were generated by using a computerized dose-planning system (GammaPlan PFX, Elekta Instruments $\mathrm{AB})$ in which gadolinium-enhanced axial three-dimensional T1-magnetization-prepared rapid acquisition gradient echo magnetic resonance (MR) images (1.5-mm slice thickness) were fused with CT images (1.25-mm slice thickness). The image difference between the non-contrast $\mathrm{T} 1$ image and the contrast-enhanced T1 MR image was used to delineate the tumor core. The outlined target encompassed the entire tumor, which was distinguished from the surrounding hematoma by use of the contrast-enhanced T1 and T2 MR images (Fig. 1). No margin was added to the planning target volume.

The treatments were usually designed to deliver $50 \%$ of the maximal dose to the margins of the target in a single fraction. The dose prescription was determined in accordance with the protocol of the Radiation Oncology Group ${ }^{21)}$, with small modifications based on factors such as volume, number, and
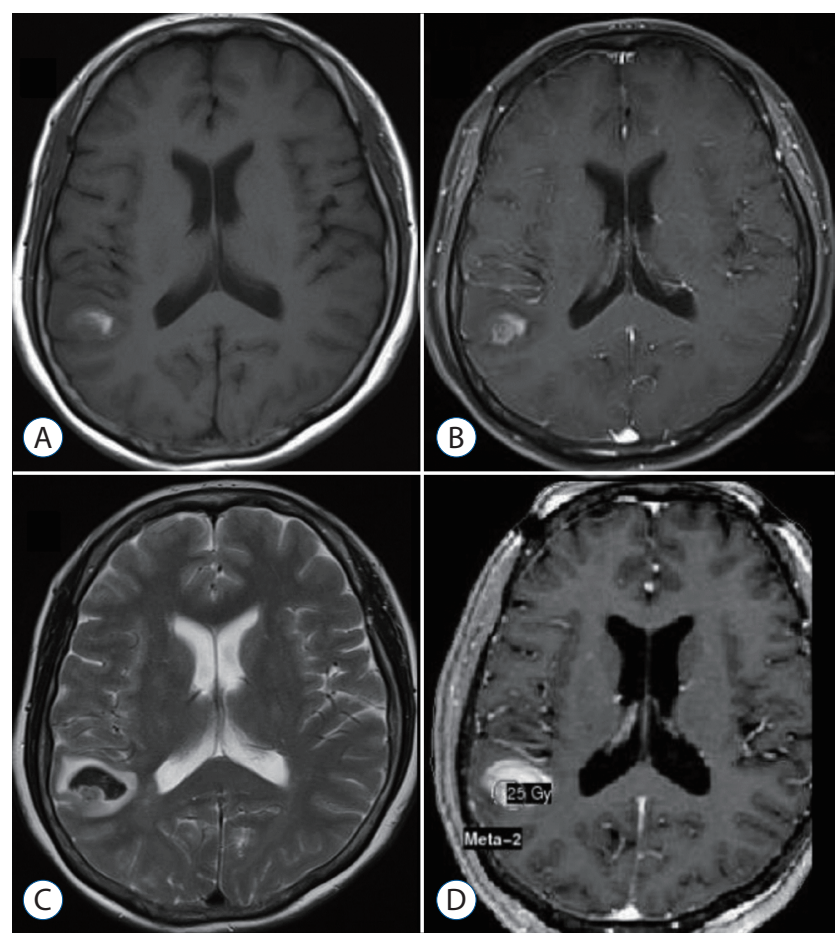

Fig. 1. Radiosurgical targeting technique for metastatic brain tumor with hemorrhage. T1-weighted (A), Gd-enhanced T1-weighted (B), and T2-weighted (C) magnetic resonance images (MRI) obtained before Gamma Knife surgery (GKS). D : Gd-enhanced T1-weighted images demonstrating dose planning. 
location of lesions, prior radiation therapy, and the predicted dose-response relationship for brain parenchymal necrosis. The most important consideration in dose prescription was total tumor volume. All patients received methylprednisolone for a short period (up to 7 days) to prevent acute brain swelling after GKS.

\section{Outcome evaluation and statistical analysis}

Follow-up clinical examinations including toxicity evaluation were performed every 1-3 months after GKS. Follow-up MRI was performed at the first clinical follow-up and every 3 months thereafter. Tumor control was assessed by comparing follow-up MRI scans with pre-GKS MRI scans. Response was classified as a complete response (tumor disappearance), a partial response (tumor volume reduction $\geq 20 \%$ ), stable disease (volume reduction $<20 \%$ or enlargement $<20 \%$ ), or disease progression (volume enlargement $\geq 20 \%$ ). A complete or partial response or stable disease at the final follow-up visit was indicative of tumor control. Distant recurrence was defined as any newly developed metastatic lesion remotely located from the previously treated lesion. Time to local recurrence was calculated from the date of GKS to the date of the first MRI that showed evidence of recurrence.

Overall survival was defined as the interval between the first GKS and death. When patient information was not available in the clinical records, a telephone interview was conducted with the patient or a family member. Overall survival was estimated by using the Kaplan-Meier method. Univariate analysis with log-rank was performed to identify predictive factors associated with the absence of local failure. $p<0.05$ was considered statistically significant. All statistical analyses were performed by using Statistical Product and Service Solution software (SPSS version 21, IBM Corp., Armonk, NY, USA).

\section{RESULTS}

\section{Patient characteristics}

During the study period, 13 patients with hemorrhagic MBTs were treated via GKS using our targeting technique. The characteristics of the patients are presented in Table 1. There were 10 males and three females, women, with a median age of 57 years (range, 31-78). The presenting symptoms included headache with or without dizziness $(n=5,38 \%)$, weakness ( $\mathrm{n}=5,38 \%)$, headache with nausea and vomiting $(\mathrm{n}=1,8 \%)$, visual disturbances $(\mathrm{n}=1,8 \%)$, and speech deficits

Table 1. Summary of patients' characteristics and treatment parameters

\begin{tabular}{lc}
\hline Characteristic & Value \\
\hline Patients & 13 \\
Gender & \\
Male & $10(77)$ \\
Female & $3(23)$ \\
Age (years) & $57(31-78)$ \\
$\leq 65$ & $9(69)$ \\
$>65$ & $4(31)$ \\
Presenting symptoms & \\
Headache (with/without dizziness) & $5(38)$ \\
Weakness & $5(38)$ \\
Headache (with nausea and vomiting) & $1(8)$ \\
Visual change & $1(8)$ \\
Speech deficits & $1(8)$ \\
KPS score & \\
$\geq 70$ & $8(62)$ \\
$<70$ & $5(38)$
\end{tabular}

\begin{tabular}{lc} 
RTOG RPA class & $1(8)$ \\
III & $7(54)$ \\
Primary tumor & $5(38)$ \\
Liver & \\
Melanoma & $7(54)$ \\
Lung & $2(15)$ \\
Kidney & $2(15)$ \\
Stomach & $1(8)$ \\
Location & $1(8)$ \\
Supratentorial & \\
Frontal & 13 \\
Temporal & $5(38)$ \\
Parietal & $0(0)$ \\
Occipital & $5(38)$ \\
Hematomattumor size in volume $\left(\mathrm{cm}^{3}\right)$ & $3(24)$ \\
Tumor size in volume $\left(\mathrm{cm}^{3}\right)$ & $25.54(10.02-46.02)$ \\
Marginal does prescribed (Gy) & $4.00(0.74-11.00)$ \\
\hline
\end{tabular}

Values are presented as median (range) or number (\%). RTOG RPA : the Radiation Therapy Oncology Group recursive partitioning analysis, KPS : Karnofsky performance status 


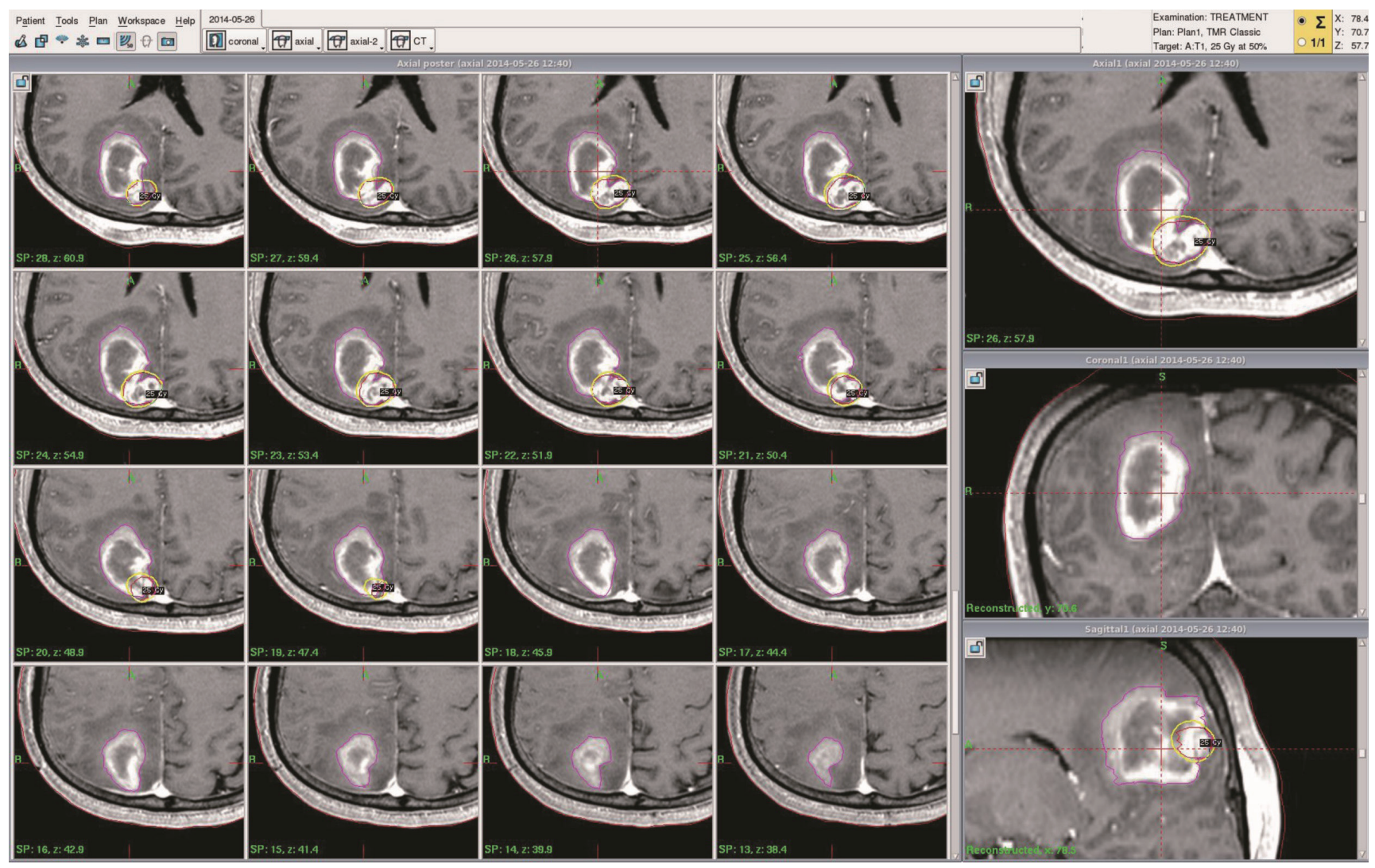

Fig. 2. The measuring method using the GammaPlan treatment planning system for tumor and hematoma volumes.

$(\mathrm{n}=1,8 \%)$. At the time of presentation, $54 \%$ of the patients had achieved disease control at the primary site, and 92\% had developed extracranial metastases. Seven (54\%) patients were recursive partitioning analysis class 2 . The primary sites of the MBTs included the liver $(n=7)$, lung $(n=2)$, kidney $(n=1)$, and stomach $(n=1)$; in two cases, the primary tumor was a melanoma. Eleven patients had solitary hemorrhagic metastatic tumors, and two patients had multiple tumors. All tumors were located in the supratentorial area.

\section{Radiosurgical treatment characteristics}

All patients underwent GKS approximately 2 weeks after tumor bleeding began to allow the patient's condition to stabilize. During the planning process, the tumor and hematoma volumes were measured by using the GammaPlan treatment planning system (Fig. 2). The average total volume (tumor volume +hematoma volume) was $25.54 \mathrm{~cm}^{3}$ (range, 10.0246.02). After excluding the hematoma volume, the mean planning target volume was $4.00 \mathrm{~cm}^{3}$ (range, 0.74-11.0). The median marginal dose prescribed was 23 Gy (range, 20-25).

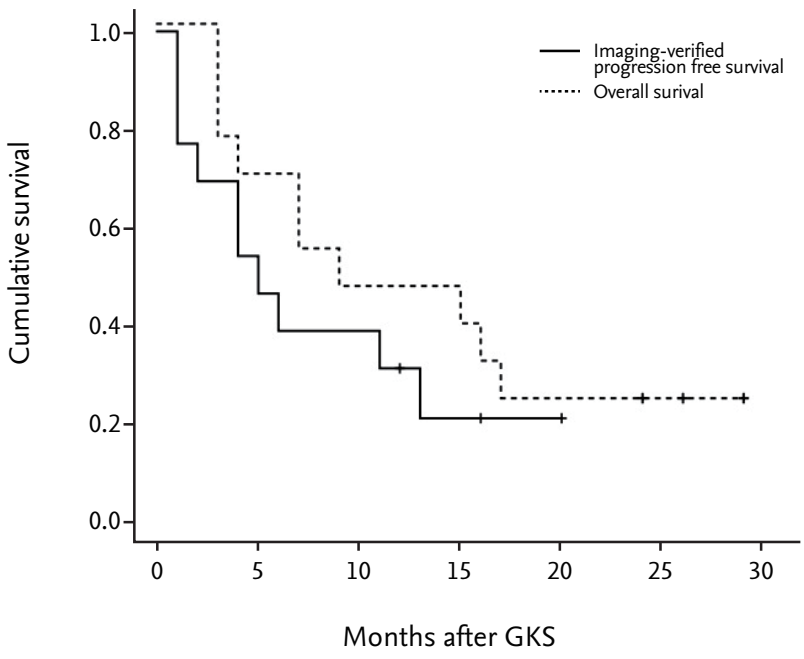

Fig. 3. Kaplan-Meier curves showing patient's overall survival and imaging-verified progression free survival. The median overall survival time after GKS was 12.5 months at the time of evaluation. The actuarial survival rates were $69.2 \%$ and $46.1 \%$ at 6 and 12 months after GKS, respectively. Imaging-verified progression free survival rates were $46.1 \%$ and $30.7 \%$ at 6 and 12 months after GKS, respectively. GKS : Gamma Knife surgery. 


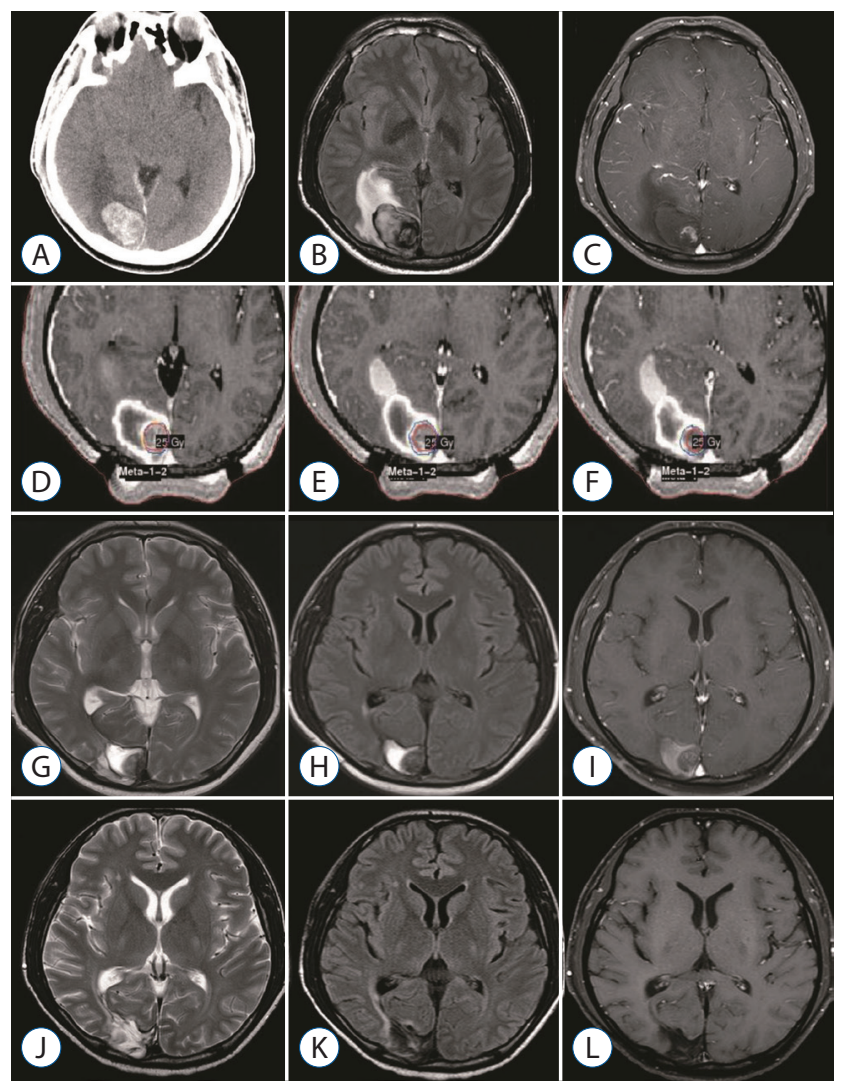

Fig. 4. A 40-year-old man with hepatocellular carcinoma presented with visual disturbance and headache. Brain computed tomography (CT) and magnetic resonance imaging revealed metastatic brain tumor with hemorrhage. GKS was performed with $25 \mathrm{~Gy}$ at the $50 \%$ isodose line. At the time of treatment, the hematoma and tumor volume measured 22.17 $\mathrm{cm}^{3}$ and $2.50 \mathrm{~cm}^{3}$, respectively. The serial images document success in the local tumor control. CT (A), FLAIR (B), and Gd-enhanced T1-weighted (C) images obtained before GKS. Gd-enhanced T1-weighted images demonstrating dose planning (D-F). T2-weighted (G), FLAIR (H), and Gdenhanced T1-weighted (I) images obtained 2 months after GKS. T2weighted (J), FLAIR (K), and Gd-enhanced T1-weighted (L) images obtained 15 months after GKS. GKS : Gamma Knife surgery, FLAIR : fluid attenuated inversion recovery.

\section{Tumor control, overall survival, and toxicity}

The median follow-up period for all patients was 12.5 months (range, 3-29). During the follow-up period, all patients were evaluated via MRI to assess tumor control. The median image follow-up period was 6.3 months (range, 1-20). Local tumor control was achieved in 12 (92\%) cases (tumor disappearance $23 \%$, tumor regression $46 \%$, and stable disease $23 \%$ ). Local recurrence in the solid portion of the tumor was observed in one patient (8\%) 11 months after GKS. Distant recurrence was observed in five patients (38\%). The patients with local or distant recurrence received salvage therapy along
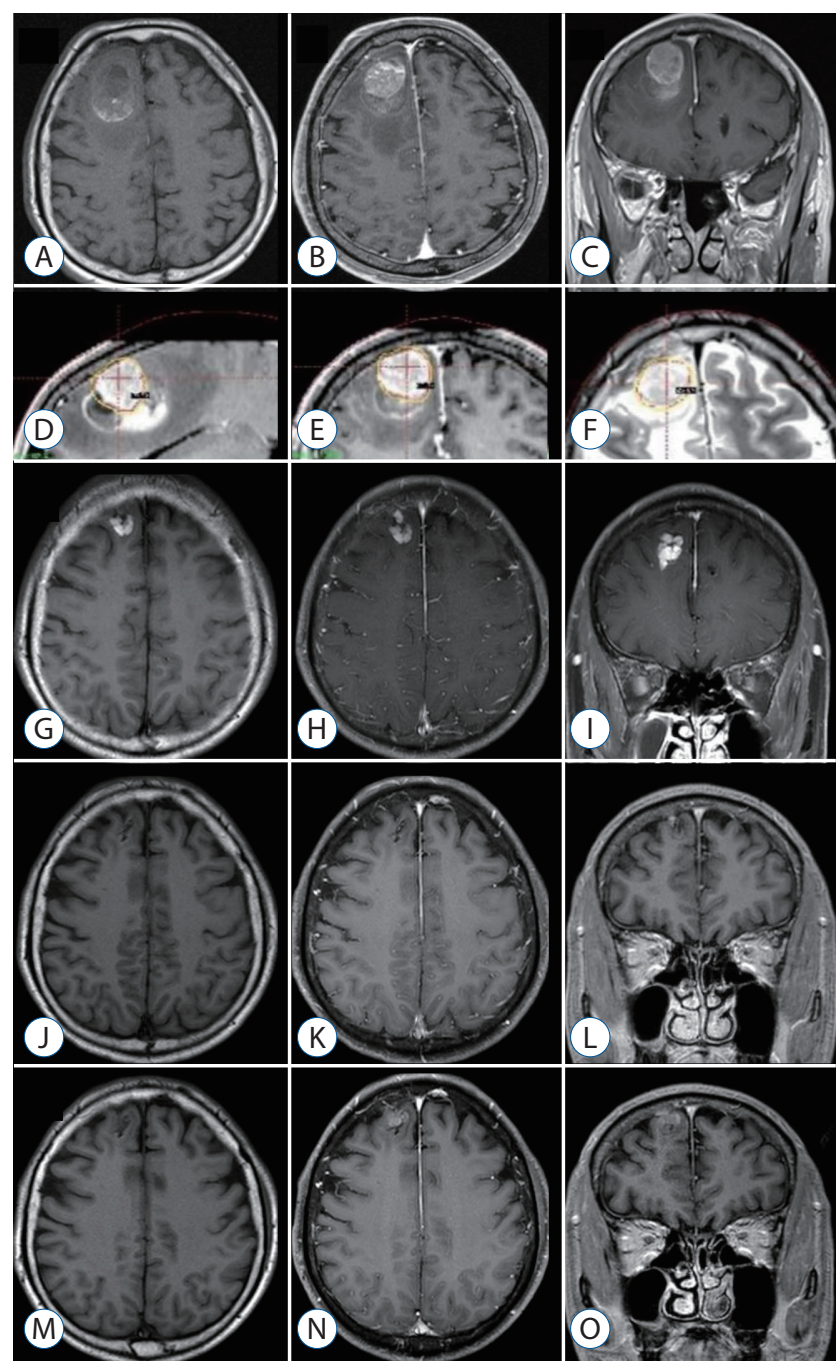

Fig. 5. A 63-year-old man with small-cell lung carcinoma presented with headache with dizziness. Brain MRI showed metastatic brain tumor with hemorrhage. GKS was performed with $25 \mathrm{~Gy}$ at the $50 \%$ isodose line. At the time of treatment, the hematoma and tumor volume measured 12.15 $\mathrm{cm}^{3}$ and $7.10 \mathrm{~cm}^{3}$, respectively. The serial images document success in the local tumor control until 9 months of follow-up. However, the recurred tumor was detected in previously treated area of the solid tumor portion on the follow-up MRI at 11 month follow-up. -weighted axial (A), Gd-enhanced T1-weighted axial (B), and coronal (C) images obtained before GKS. Gd-enhanced T1-weighted and T2-weighted images demonstrating dose planning (D-F). G-I : T1-weighted axial (A), Gd-enhanced T1-weighted axial (B), and coronal (C) images obtained 3 months after GKS. J-L : T1-weighted axial (A), Gd-enhanced T1-weighted axial (B), and coronal (C) images obtained 9 months after GKS. M-O : T1weighted axial (A), Gd-enhanced T1-weighted axial (B), and coronal (C) images obtained 11 months after GKS. MRI : magnetic resonance imaging, GKS: Gamma Knife surgery.

with further radiosurgery.

Univariate analysis was used to identify potential factors associated with the absence of local failure. No factor reached 
statistical significance.

The figure demonstrates patient's overall survival and imaging-verified progression free survival (Fig. 3). The median overall survival time after GKS was 12.5 months at the time of evaluation. The actuarial survival rates were $69.2 \%$ and $46.1 \%$ at 6 and 12 months after GKS, respectively. Imaging-verified progression free survival rates were $46.1 \%$ and $30.7 \%$ at 6 and 12 months after GKS, respectively. Three patients are still alive at the time of this review. To date, 10 patients included in this series have died, one from progressive central nervous system disease and the others from progressive systemic disease. No patients developed any new neurologic deficits after GKS, and no grade 3 or higher toxicity was observed.

\section{Illustrative cases}

\section{Case 1 (MBT with hemorrhage, GKS targeting at only tumor core)}

A 40-year-old man with hepatocellular carcinoma presented with visual disturbance and headache. Brain CT and MRI showed an enhancing mass with hemorrhage, compatible with metastatic brain tumor (Fig. 4A-C). After waiting the patient's stabilization for 2 weeks, GKS with a marginal dose of 25 Gy was performed for MBTs via our radiosurgical planning technique (the radiosurgical target was confined to the enhancing tumor core) (Fig. 4D-F). The measured hematoma volume and tumor volume were $22.17 \mathrm{~cm}^{3}$ and $2.50 \mathrm{~cm}^{3}$, respectively. After GKS, the patient was gradually recovered without complication (Fig. 4G-I). On follow-up MRI 15 month later, the treated tumor was well controlled without local recurrence (Fig. 4J-L).

\section{Case 2 (recurred tumor after GKS using our radiosur- gical technique)}

A 63-year-old man with small-cell lung carcinoma presented with headache with dizziness. Brain MRI showed a metastatic brain tumor with hemorrhage (Fig. 5A-C). After waiting the patient's stabilization for two weeks, GKS with a marginal dose of 25 Gy was performed with our radiosurgical technique (Fig. 5D-F). The measured hematoma volume and tumor volume were $12.15 \mathrm{~cm}^{3}$ and $7.10 \mathrm{~cm}^{3}$, respectively. On follow-up MRI at 3 months follow-up, the treated tumor was significantly regressed (Fig. 5G-I). After that, the tumor was disappeared for the following 6-month periods (Fig. 5J-L). However, the recurred tumor was detected in previously treated area of the solid tumor portion on the follow-up MRI at 11 monthsfollow-up (Fig. 5M-O). Consequently, he was re-treated with GKS for the recurred tumor.

\section{DISCUSSION}

Owing to advances in radiosurgical techniques and the accumulation of radiosurgical experiences, the number of indications for radiosurgery for treatment of MBTs has increased and continues to increase ${ }^{17,23)}$. Before the late 1900s, the combination of whole-brain radiotherapy (WBRT) and corticosteroid administration was the mainstay for management of MBTs; since that time, surgical treatment has moved to the forefront, along with improvements in operative neurosurgery and brain imaging modalities such as CT and $\mathrm{MRI}^{3,14,18)}$. Several randomized prospective clinical trials have validated the usefulness of surgical treatment for treatment of MBTs ${ }^{15)}$. More recently, an innovative radiation treatment with focused high-energy radiation, termed stereotactic radiosurgery (SRS), was developed and applied to clinical practice. In last two decades, a tremendous number of patients with MBTs have been treated via SRS mainly by using a Gamma Knife system or less common linear accelerator ${ }^{2)}$. Although no high-powered randomized controlled studies have compared the effectiveness of SRS with that of standard surgical resection, the superiority of SRS compared with WBRT, in terms of long-term tumor control and reduced post-radiation toxicity of cognitive function, is well established ${ }^{5,6,8,11,13,20)}$. Consequently, in recent years, the widely used oncology guidelines have recommended SRS as a primary treatment option for patients with more than three MBTs ${ }^{17)}$.

This study describes our experience in the use of GKS for treatment of MBTs with exophytic hemorrhage. We reviewed our results, focusing on efficiency in tumor control and treatment toxicity. The local tumor control rates for MBTs treated via SRS range from approximately $71 \%$ to $96 \%$ in general pathology ${ }^{10)}$ and $49 \%$ to $97 \%$ in radio-resistant pathology ${ }^{24,25)}$. Among the radio-resistant pathologies, melanomas have been shown to respond less effectively to SRS than do renal cell carcinomas and more effectively than do hepatocellular carcinomas $^{19,25)}$. Our results showed a $92 \%$ tumor control rate for hemorrhagic MBTs, and no GKS-related complications were 
observed during the follow-up period.

Although our series contained a high percentage (77\%) of tumors with radio-resistant histology, including hepatocellular carcinomas, we obtained a similar or modestly higher tumor control rate than did previous studies. This presumably reflects the radiation dose used and the shortness of the follow-up period. We chose a relatively high radiation dose (median, 23 Gy) owing to the radiation-resistant histology of most of the tumors in our series. Nevertheless, we achieved good tumor control without treatment toxicity. The short follow-up duration in our study (median, 12.5 months) may limit the interpretation of our results. However, six of the 13 the patients were followed-up for more than 1 year, one (17\%) of whom experienced local treatment failure. This result is similar to previous radiosurgical and surgical results for $\mathrm{MBTs}^{15,26)}$.

When radiologically treating hemorrhagic MBTs, we used a radiosurgical targeting technique that confines the tumor core within the hematoma. Using this approach, we experienced one case of local recurrence, which occurred in the solid portion of the tumor but not at the hematoma site. It is generally accepted that MBTs require angiogenesis for sustained growth, as well as vessel incorporation, at least in the initial phase of tumor progression, for vascularization ${ }^{7}$. An immunohistochemical analysis in a mouse model showed that tumor cells located less than $100 \mathrm{~mm}$ from a blood vessel are viable, whereas more distant tumor cells are apoptotic ${ }^{9)}$. Presumably, the scattered tumor cells in hematomas have a lower growth activity than do the cells in the solid tumor owing to the inadequate microenvironment of hematomas. Additionally, we performed GKS after waiting for the acute period about two weeks because of the possibility of serious situation needed surgery. We believe our policy (delayed radiosurgery after waiting for the acute period) has benefits considering patient's safety and treatment efficacy. In the perspective of planning image, it was easier to delineate the tumor from surrounding hematoma in our experience. In process of time, the imaging differences between surrounding hematoma and solid tumor become more prominent, which is caused by changed MR images of surrounding hematoma based on time period and more prominently shown the enhancing solid tumor. Therefore, radiosurgery could be performed in the early period after tumor bleeding according to patients' neurological status and hemorrhage volume.

Hemorrhage is often associated with MBTs. The percentage of hemorrhage is varied, but typically influenced by primary cancer $^{1)}$. One pathology-based report showed that intra-tumoral hemorrhage developed in 24\% (36/147) of MBTs ${ }^{12)}$. In the light of management, there is no difference between hemorrhagic MBTs and non-hemorrhagic MBTs ${ }^{17)}$. Some reported that surgery has the merits in the management of hemorrhagic MBTs because of following reasons ${ }^{26)}$. Firstly, hemorrhagic MBTs have a good dissection plane, so it is relatively easy to remove them completely. Secondly, hemorrhage might cause the disruption of the blood supply to the tumor and subsequent tumor cell necrosis. Nevertheless, the innate features of MBTs like local and distant recurrence require adjuvant treatment along with surgery ${ }^{22)}$. Recently, the role of SRS for the management of MBTs has increased as primary or adjuvant treatment ${ }^{17)}$. With the advances of multimodal therapy in cancer management, the expansion of their role is expected. So, it is necessary to develop the new radiosurgical technique to extend and improve their role. We hypothesized that our radiosurgical planning technique would efficiently reduce radiation toxicity and result in oncologic control. Our results demonstrate overall safety and acceptable local tumor control and therefore support the use of this novel approach for the treatment of hemorrhagic MBTs. GKS has many advantages in patients with advanced malignancy, most notably, its capacity to treat lesions that are not amenable to surgical resection; moreover, unlike open surgery, it can be performed in an outpatient setting. Given the emergence of novel systemic therapies and resultant improvements in the survival of patients with advanced malignancies, GKS in conjunction with our technique is a promising option for patients with hemorrhagic MBTs.

\section{CONCLUSION}

Our experience shows that GKS performed in conjunction with our targeting technique safely and effectively treats hemorrhagic MBTs. The success of this technique may reflect the presence of scattered metastatic tumor cells in the hematoma that do not proliferate owing to the inadequate microenvironment of the hematoma. GKS using only-tumor-targeting technique seems to be a useful treatment option in selected patients with hemorrhagic MBTs. To establish a solid conclusion, further study with a larger cohort of hemorrhagic MBTs is necessary. 


\section{CONFLICTS OF INTEREST}

The authors have no financial conflicts of interest.

\section{INFORMED CONSENT}

Informed consent was obtained from all individual participants included in this study.

\section{- Acknowledgments}

We express great thanks to Hyung Jung Kim, RN, for collecting data on the patients. This work was supported by a National Research Foundation of Korea (NRF) grant, funded by the Ministry of Education (No. 2017R1C1B5018208).

\section{References}

1. Aghi MK, Ogilvy CS, Carter BS : Ch.69 Surgical management of intracerebral hemorrhage : Schmidek \& sweet operative neurosurgical techniques: indications, methods, and results, ed 6. Philadelphia : Saunders, 2012, pp825

2. Ajay Niranjan JNJ, Hideyuki Kano, Douglas Kondziolka, L. Dade Lunsford, John C. Flickinger : Ch.256 Gamma Knife radiosurgery : Youmans Neurological Surgery, ed 6. Philadelphia : Saunders, 2011, pp2633-2634

3. Al-Shamy G, Sawaya R : Management of brain metastases: the indispensable role of surgery. J Neurooncol 92 : 275-282, 2009

4. Alexandru D, Bota DA, Linskey ME : Epidemiology of central nervous system metastases. Prog Neurol Surg 25 : 13-29, 2012

5. Andrews DW, Scott CB, Sperduto PW, Flanders AE, Gaspar LE, Schell $M C$, et al. : Whole brain radiation therapy with or without stereotactic radiosurgery boost for patients with one to three brain metastases: phase III results of the RTOG 9508 randomised trial. Lancet 363 : 1665-1672, 2004

6. Aoyama H, Shirato H, Tago M, Nakagawa K, Toyoda T, Hatano K, et al. : Stereotactic radiosurgery plus whole-brain radiation therapy vs stereotactic radiosurgery alone for treatment of brain metastases: a randomized controlled trial. JAMA 295 : 2483-2491, 2006

7. Bugyik E, Dezso K, Reiniger L, László V, Tóvári J, Tímár J, et al. : Lack of angiogenesis in experimental brain metastases. J Neuropathol Exp Neurol 70 : 979-991, 2011

8. Chang EL, Wefel JS, Hess KR, Allen PK, Lang FF, Kornguth DG, et al. Neurocognition in patients with brain metastases treated with radiosurgery or radiosurgery plus whole-brain irradiation: a randomised controlled trial. Lancet Oncol 10 : 1037-1044, 2009

9. Fidler IJ, Yano S, Zhang RD, Fujimaki T, Bucana CD : The seed and soil hypothesis: vascularisation and brain metastases. Lancet Oncol 3 :
53-57, 2002

10. Gregory JA, Murad WAF : Ch.258 Radiosurgery of malignant tumors : Youmans Neurological Surgery, ed 6. Philadelphia : Saunders, 2011, pp2645

11. Kocher M, Soffietti R, Abacioglu U, Villa S, Fauchon F, Baumert BG, et al. : Adjuvant whole-brain radiotherapy versus observation after radiosurgery or surgical resection of one to three cerebral metastases: results of the EORTC 22952-26001 study. J Clin Oncol 29 : 134-141, 2010

12. Kondziolka D, Bernstein $\mathrm{M}$, Resch $\mathrm{L}$, Tator $\mathrm{CH}$, Fleming JR, Vanderlinden $R G$, et al. : Significance of hemorrhage into brain tumors: clinicopathological study. J Neurosurg 67 : 852-857, 1987

13. Kondziolka D, Patel A, Lunsford LD, Kassam A, Flickinger JC : Stereotactic radiosurgery plus whole brain radiotherapy versus radiotherapy alone for patients with multiple brain metastases. Int J Radiat Oncol Biol Phys 45 : 427-434, 1999

14. Lee EQ : Nervous system metastases from systemic cancer. Continuum (Minneap Minn) 21(2 Neuro-oncology) : 415-428, 2015

15. Mut M : Surgical treatment of brain metastasis: a review. Clin Neurol Neurosurg $114: 1-8,2012$

16. Nabors LB, Ammirati M, Bierman PJ, Brem H, Butowski N, Chamberlain MC, et al. : Central nervous system cancers. J Natl Compr Canc Netw 11 : 1114-1151, 2013

17. Nabors LB, Portnow J, Ammirati M, Brem H, Brown P, Butowski $N$, et al. : Central nervous system cancers, version 2.2014. Featured updates to the NCCN Guidelines. J Natl Compr Canc Netw 12 : 1517-1523, 2014

18. Owonikoko TK, Arbiser J, Zelnak A, Shu HK, Shim H, Robin AM, et al. : Current approaches to the treatment of metastatic brain tumours. Nat Rev Clin Oncol 11 : 203-222, 2014

19. Park ES, Kwon DH, Park JB, Lee DH, Cho YH, Kim JH, et al. : Gamma Knife surgery for treating brain metastases arising from hepatocellular carcinomas. J Neurosurg 121 Suppl : 102-109, 2014

20. Patil CG, Pricola K, Sarmiento JM, Garg SK, Bryant A, Black KL : Whole brain radiation therapy (WBRT) alone versus WBRT and radiosurgery for the treatment of brain metastases. Cochrane Database Syst Rev (9) : CD006121, 2012

21. Shaw E, Scott C, Souhami L, Dinapoli R, Kline R, Loeffler J, et al. : Single dose radiosurgical treatment of recurrent previously irradiated primary brain tumors and brain metastases: final report of RTOG protocol 90 05. Int J Radiat Oncol Biol Phys 47 : 291-298, 2000

22. Soon YY, Tham IW, Lim KH, Koh WY, Lu JJ : Surgery or radiosurgery plus whole brain radiotherapy versus surgery or radiosurgery alone for brain metastases. Cochrane Database Syst Rev (3) : CD009454, 2014

23. Suh JH : Stereotactic radiosurgery for the management of brain metastases. N Engl J Med 362 : 1119-1127, 2010

24. Yaeh A, Nanda T, Jani A, Rozenblat T, Qureshi Y, Saad S, et al. : Control of brain metastases from radioresistant tumors treated by stereotactic radiosurgery. J Neurooncol 124 : 507-514, 2015

25. Yen $C P$, Steiner $L$ : Ch.5 Gamma Knife surgery for cerebral vascular malformations and tumors: Schmidek \& Sweet operative neurosurgical techniques: indications, methods, and results, ed 6 . Philadelphia : Saunders, 2012, pp77-78

26. Yoo H, Jung E, Gwak HS, Shin SH, Lee SH : Surgical outcomes of hemorrhagic metastatic brain tumors. Cancer Res Treat 43 : 102-107, 2011 